\title{
Some higher norm inequalities for composition of power operators
}

Huacan $\mathrm{Li}^{1 *}$ and Qunfang $\mathrm{Li}^{2}$

"Correspondence: hcli@jxust.edu.cn ${ }^{1}$ School of Science, JiangXi University of Science and Technology, Ganzhou, China Full list of author information is available at the end of the article

\begin{abstract}
In this paper, we first prove the local $L^{s}$ norm estimate of composite operators $\Delta^{k} G^{m}(u)$ by use of the $L^{s}$ norm of $u$. Then we establish the local and global higher norm inequalities of $\Delta^{k} G^{m}(u)$. Simultaneously, we also give a global higher norm estimate with Radon measure. Finally, as applications of these results, we give two examples to estimate the higher norm of $\Delta^{k} G^{m}(u)$.
\end{abstract}

Keywords: Power operators; Radon measure; Whitney cover; Higher norm inequalities

\section{Introduction}

In this paper, our purpose is investigating some higher norm inequalities for composition of power operators $\triangle^{k} G^{m}$ on a bounded domain $M$, where $k, m$ are positive integers, $\triangle$ is Laplace-Beltrami operator, and $G$ is Green's operator. The norm estimate of operators applied to differential forms is an important and interesting research topic in some areas of mathematic analysis and has achieved fruitful results; see [1-11] for more detail. Some these results improved the development of some other branches of mathematics and mathematical physics; see [12-18] for details. In previous related research about norm estimates of operators the study mostly concentrated on estimates of the $L^{t}$ norm of operators and applying them to differential forms in terms of the $L^{t}$ norm of differential forms. Therefore, if $s>t$, then we could not estimate the $L^{s}$ norm of operators by the $L^{t}$ norm of differential forms from the literature. This motivated us to research the higher norm of operators than of differential forms. Since the norm estimate of a composite operator is more complicated than that of a single operator, in this paper, we choose the composition of power operators $\Delta^{k} G^{m}$ to be the research object. In this paper, we first give the local $L^{s}$ norm estimate of $\triangle^{k} G^{m}(u)$ by the $L^{s}$ norm of $u$ in Theorem 2.5. Then based on Theorem 2.5, we prove the local and global higher norm inequalities of $\Delta^{k} G^{m}(u)$ separately presented in Theorems 2.6-2.8 and 3.2. Simultaneously, we also establish the global higher norm estimate with Radon measure in Theorem 3.3. Finally, we give two examples as applications of Theorem 3.2.

We start this paper by introducing some notations and definitions in [19]. Let $M \subset R^{n}$ $(n \geq 2)$ be a bounded domain, $B$ be a ball, and $\sigma B$ be the ball with the same center as

(c) The Author(s) 2020. This article is licensed under a Creative Commons Attribution 4.0 International License, which permits use, sharing, adaptation, distribution and reproduction in any medium or format, as long as you give appropriate credit to the original author(s) and the source, provide a link to the Creative Commons licence, and indicate if changes were made. The images or other third party material in this article are included in the article's Creative Commons licence, unless indicated otherwise in a credit line to the material. If material is not included in the article's Creative Commons licence and your intended use is not permitted by statutory regulation or exceeds the permitted use, you will need to obtain permission directly from the copyright holder. To view a copy of this licence, visit http://creativecommons.org/licenses/by/4.0/. 
$B$ satisfying $\operatorname{diam}(\sigma B)=\sigma \operatorname{diam}(B)$. We do not distinguish balls from cubes in this paper. By $\Lambda^{k}=\Lambda^{k}\left(R^{n}\right)(k=1,2, \ldots, n)$ we denote the linear space of all $k$-forms $u(x)=$ $\sum_{I} u_{I}(x) d x_{I}=\sum u_{i_{1} i_{2} \cdots i_{k}} d x_{i_{1}} \Lambda d x_{i_{2}} \Lambda \cdots \Lambda d x_{i_{k}}$ with summation over all ordered $k$-tuples $I=\left(i_{1}, i_{2}, \ldots, i_{k}\right), 1 \leq i_{1}<i_{2}<\cdots<i_{k} \leq n, k=1,2, \ldots, n$. If the coefficient $u_{I}(x)$ of $k$-forms is differentiable on $M$, then we call $u(x)$ a differential $k$-form on $M$. As usual, we use $C^{\infty}\left(M, \Lambda^{k}\right)$ to denote the space of smooth $k$-forms in a domain $M, D^{\prime}\left(M, \Lambda^{k}\right)$ to denote the space of all differential $k$-forms. Let $L^{p}\left(M, \Lambda^{k}\right)$ be the set of differential $k$-forms $u(x)=\sum_{I} u_{I}(x) d x_{I}$ on $M$ with $\int_{M}\left|u_{I}\right|^{p}<\infty$ for all ordered $k$-tuples $I$. The norm of a $k$ form $u(x)$ on $M$ is defined by

$$
\|u(x)\|_{p, M}=\left(\int_{M}|u(x)|^{p} d x\right)^{\frac{1}{p}}=\left(\int_{M}\left(\sum_{I}\left|u_{I}(x)\right|^{2}\right)^{\frac{p}{2}} d x\right)^{\frac{1}{p}},
$$

and then $L^{p}\left(M, \Lambda^{k}\right)$ is a Banach space. As usual, we use $\star$ to denote the Hodge star operator and $\operatorname{dist}(x, M)$ to denote the distance of the point $x$ from the set $M$. Also, we use $d: D^{\prime}\left(M, \Lambda^{k}\right) \rightarrow D^{\prime}\left(M, \Lambda^{k+1}\right)$ to denote the differential operator and $d^{\star}: D^{\prime}\left(M, \Lambda^{k+1}\right) \rightarrow$ $D^{\prime}\left(M, \Lambda^{k}\right)$ to denote the Hodge codifferential operator defined by $d^{\star}=(-1)^{n k+1} \star d \star$ on $D^{\prime}\left(M, \Lambda^{k+1}\right)$. The $n$-dimensional Lebesgue measure of a set $E \subseteq R^{n}$ is denoted by $|E|$. For any differential form $u$, the average of $u$ over $B$ is defined as $u_{B}=\frac{1}{|B|} \int_{B} u d x$. All integrals involved in this paper are the Lebesgue integrals. The Laplace-Beltrami operator $\triangle$ is defined by $\Delta=d d^{\star}+d^{\star} d$. We define Green's operator $G$ on the space of smooth $k$-forms in $M$ by setting $G(u)$ to be a solution of Poisson's equation $\triangle G(u)=u-H(u)$, where $H$ is the harmonic projection; see $[1,7,19-22]$ for more detail about the Laplace-Beltrami operator $\triangle$, Green's operator $G$, and projection operator $H$. We call $w$ a weight if $w \in L_{\text {loc }}^{1}\left(R^{n}\right)$ and $w>0$ a.e. For any Radon measure $v$ defined by $d v=w(x) d x$, we define the $L^{p}$-norm of a measurable function $f$ with Radon measure over $M$ by

$$
\|f\|_{p, M, v}=\left(\int_{M}|f|^{p} d v\right)^{\frac{1}{p}}=\left(\int_{M}|f|^{p} w(x) d x\right)^{\frac{1}{p}}
$$

and the Radon measure of $E$ by $v(E)=\int_{E} d \nu=\int_{E} w(x) d x$.

The nonlinear partial differential equation

$$
d^{\star} A(x, d u)=0
$$

is called the $A$-harmonic equation, where $A: M \times \Lambda^{k}\left(R^{n}\right) \rightarrow \Lambda^{K}\left(R^{n}\right)$ satisfies the following conditions:

$$
|A(x, \xi)| \leq a|\xi|^{p-1} \quad \text { and } \quad A(x, \xi) \xi \geq|\xi|^{p}
$$

for almost every $x \in M$ and all $\xi \in \Lambda^{k}\left(R^{n}\right)$, where $a>0$ is a constant, and $1<p<\infty$ is a fixed exponent associated with (1.3). For more details about $A$-harmonic equation, see $[3,4,8,19,23,24]$.

\section{Local higher norm inequalities for $\Delta^{k} G^{m}(u)$}

In this section, we first give the $L^{s}$ norm estimate of $\triangle^{k} G^{m}(u)$ in terms of the $L^{s}$ norm of $u$. Then based on this result, we establish local higher norm inequalities for $\triangle^{k} G^{m}(u)$ in two cases. 
Let $\psi$ be a strictly increasing convex function on $[0,+\infty)$ with $\psi(0)=0$, and let $u$ be a differential form on a bounded domain $M$. Then $\psi\left(\kappa|u|+\left|u_{M}\right|\right) \in L^{1}(M, v)$ for any real number $\kappa>0$ and $v\left(\left\{x \in M:\left|u-u_{M}\right|>0\right\}\right)>0$, where $v$ is the Radon measure defined by $d v=w(x) d x$ for a weight $w(x)$. For any positive constant $a$, we have

$$
\int_{M} \psi\left(\frac{1}{2}\left|u-u_{M}\right|\right) d v \leq C_{1} \int_{M} \psi(a|u|) d v \leq C_{2} \int_{M} \psi\left(2 a\left|u-u_{M}\right|\right) d v
$$

for some constants $C_{1}>0$ and $C_{2}>0$. Let $\psi(u)=u^{s}, s>1, w(x)=1$, and let $M$ be a ball $B$ in this inequality. Then there exist two positive constants $C_{3}$ and $C_{4}$, independent of $u$, such that

$$
\left\|u-u_{B}\right\|_{s, B} \leq C_{3}\|u\|_{s, B} \leq C_{4}\left\|u-u_{B}\right\|_{s, B}
$$

for all balls $B$ with $\left|\left\{x \in B:\left|u-u_{B}\right|>0\right\}\right|>0$. Inequality (2.1) indicates the norm $\left\|u-u_{B}\right\|_{s, B}$ comparable to the norm $\|u\|_{s, B}$.

Lemma 2.1 ([5]) Let $u \in C^{\infty}(M, \Lambda)$ be a smooth differential form defined on $M$. Then there exists a constant $C=C(s)$, independent of $u$, such that

$$
\left\|d d^{\star} G(u)\right\|_{s, B}+\left\|d^{\star} d G(u)\right\|_{s, B}+\|d G(u)\|_{s, B}+\left\|d^{\star} G(u)\right\|_{s, B}+\|G(u)\|_{s, B} \leq C(s)\|u\|_{s, \sigma B}
$$

for any ball $B$ with $\sigma B \subset M$, where $\sigma>1$ and $1<s<\infty$ are constants.

Lemma 2.2 ([25]) Let u be a solution of A-harmonic equation (1.3) in a domain M. Then there exists a constant $C$, independent of $u$, such that

$$
\|u\|_{s, B} \leq C|B|^{(t-s) / s t}\|u\|_{t, \sigma B}
$$

for all balls $B$ with $\sigma B \subset M$, where $\sigma>1$ and $0<s, t<\infty$ are constants.

Lemma 2.3 ([24]) Let $u \in D^{\prime}\left(Q, \Lambda^{l}\right)$ and $d u \in L^{p}\left(Q, \Lambda^{l+1}\right)$. Then $u-u_{Q}$ is in $L^{n p /(n-p)}\left(Q, \Lambda^{l}\right)$, and

$$
\left(\int_{Q}\left|u-u_{Q}\right|^{n p /(n-p)} d x\right)^{(n-p) / n p} \leq C_{p}(n)\left(\int_{Q}|d u|^{p} d x\right)^{1 / p}
$$

for a cube or a ball $Q$ in $R^{n}$, where $l=0,1,2, \ldots, n-1$ and $1<p<n$.

Lemma 2.4 Let $u$ be a smooth differential form defined on $M, G$ be Green's operator, and $\triangle$ be the Laplace-Beltrami operator defined by $\Delta=d d^{\star}+d \star d$. Then the Laplace-Beltrami operator $\triangle$ and Green's operator $G$ are commutable, that is,

$$
\triangle G(u)=G \triangle(u)
$$

Proof From $\left[19\right.$, p. 88] we find that Green's operator $G$ commutes with $d$ and $d^{\star}$. Therefore, for any differential form $u$, we have

$$
G d(u)=d G(u), G d^{\star}(u)=d^{\star} G(u) .
$$


From (2.3) and the definition of the Laplace-Beltrami operator $\Delta$ we obtain

$$
\begin{aligned}
\triangle G(u) & =\left(d d^{\star}+d^{\star} d\right) G(u) \\
& =d d^{\star} G(u)+d^{\star} d G(u) \\
& =G d d^{\star}(u)+G d^{\star} d(u) \\
& =G\left(d d^{\star}+d^{\star} d\right)(u) \\
& =G \triangle(u) .
\end{aligned}
$$

Thus we complete the proof of Lemma 2.4.

Now we will give the following local $L^{s}$-norm estimate of composite operator $\triangle^{k} G^{m}$, which will be used in the proof of higher norm theorems.

Theorem 2.5 Let $u \in L_{\mathrm{loc}}^{s}\left(M, \Lambda^{l}\right)(1<s<\infty, l=1,2, \ldots, n)$ be a smooth differential form defined on $M, G$ be Green's operator, and $\triangle$ be the Laplace-Beltrami operator. Then there exists a constant $C$, independent of $u$, such that

$$
\left\|\Delta^{k} G^{m}(u)\right\|_{s, B} \leq C\|u\|_{s, \sigma B}
$$

for all balls $B$ with $\sigma B \subset M$ and any positive integer $k \leq m$, where $\sigma>1$.

Proof We prove this theorem in two steps: (1) First, let $k=m$. For any differential form $u$, from Lemma 2.4 we have $G \triangle(u)=\triangle G(u)$, and thus

$$
\triangle^{k} G^{k}(u)=(\triangle G)^{k}(u)
$$

Hence (2.4) is equivalent to

$$
\left\|(\triangle G)^{k}(u)\right\|_{s, B} \leq C\|u\|_{s, \sigma B} .
$$

Now we use mathematical induction to prove (2.6). Let $k=1$. By Lemma 2.1 we have

$$
\begin{aligned}
\|\Delta G(u)\|_{s, B} & =\left\|\left(d d^{\star}+d^{\star} d\right) G(u)\right\|_{s, B} \\
& \leq\left\|d d^{\star} G(u)\right\|_{s, B}+\left\|d^{\star} d G(u)\right\|_{s, B}=C_{1}\|u\|_{s, \sigma_{1} B},
\end{aligned}
$$

where $\sigma_{1} B \subset M$ with $\sigma_{1}>1$. Assume that (2.6) holds for $k=k^{\prime}, k^{\prime}=1,2, \ldots$, that is,

$$
\left\|(\triangle G)^{k^{\prime}}(u)\right\|_{s, B} \leq C_{2}\|u\|_{s, \sigma_{2} B},
$$

where $\sigma_{2}>1$ is a constant such that $\sigma_{2} B \subset M$. Now we prove that (2.6) holds for $k=k^{\prime}+1$. From (2.7) and (2.8) we have

$$
\begin{aligned}
\left\|(\Delta G)^{k^{\prime}+1}(u)\right\|_{s, B} & =\left\|\Delta G\left((\Delta G)^{k^{\prime}}(u)\right)\right\|_{s, B} \\
& \leq C_{3}\left\|(\triangle G)^{k^{\prime}}(u)\right\|_{s, \sigma_{3} B} \leq C_{4}\|u\|_{s, \sigma_{4} B},
\end{aligned}
$$

where $\sigma_{4}>\sigma_{3}>1$ and $\sigma_{4} B \subset M$. From (2.9) it follows that if $k=m$, then (2.4) holds. 
(2) Next, let $k<m$. From $\triangle G(u)=G \triangle(u)$ we obtain

$$
\triangle^{k} G^{m}(u)=G^{m-k}\left((\triangle G)^{k}(u)\right)
$$

For any differential form $u$, from Lemma 2.1 we have

$$
\|G(u)\|_{s, B} \leq C_{5}\|u\|_{s, \sigma_{5} B}
$$

for some constant $\sigma_{5}>1$ with $\sigma_{5} B \subset M$. Using mathematical induction and (2.11),we can easily prove that

$$
\left\|G^{k^{\prime}}(u)\right\|_{s, B} \leq C_{6}\|u\|_{s, \sigma_{6} B}
$$

for any positive integer $k^{\prime}$ and some constant $\sigma_{6}>1$ such that $\sigma_{6} B \subset M$. Combining (2.10), (2.12), and (2.6), we have

$$
\begin{aligned}
\left\|\triangle^{k} G^{m}(u)\right\|_{s, B} & =\left\|G^{m-k}\left((\triangle G)^{k}(u)\right)\right\|_{s, B} \\
& \leq C_{7}\left\|(\triangle G)^{k}(u)\right\|_{s, \sigma_{6} B} \\
& \leq C_{8}\|u\|_{s, \sigma_{7} B}
\end{aligned}
$$

for some constants $\sigma_{7}>\sigma_{6}>1$ such that $\sigma_{7} B \subset M$. Estimate (2.13) shows that (2.4) holds for $k<m$. This completes the proof of Theorem 2.5.

Next, based on Theorem 2.5, we will prove local higher norm inequalities for the composite operator $\triangle^{k} G^{m}$ in two cases.

Theorem 2.6 Let $u \in L_{\mathrm{loc}}^{t}\left(M, \Lambda^{l}\right)$ be a smooth differential form on $M, G$ be Green's operator, and $\triangle$ be the Laplace-Beltrami operator, $l=1,2, \ldots, n, 1<t<n$. Then $\Delta^{k} G^{m}(u) \in$ $L_{\mathrm{loc}}^{s}\left(M, \Lambda^{l}\right)$ for any s such that $0<s<n t /(n-t)$. Moreover, there exists a constant $C$, independent of $u$, such that

$$
\left\|\Delta^{k} G^{m}(u)\right\|_{s, B} \leq C|B|^{1 / s+1 / n-1 / t}\|u\|_{t, \sigma B}
$$

for all balls $B$ with $\sigma B \subset M$ and $|B|>d_{0}$ and any positive integer $k<m$, where $\sigma>1$ and $d_{0}>0$ are constants.

Proof We prove this theorem in the following two cases: (1) First, assume that the measure $\left|\left\{x \in B:\left|\Delta^{k} G^{m}(u)-\left(\Delta^{k} G^{m}(u)\right)_{B}\right|>0\right\}\right|=0$. Then $\triangle^{k} G^{m}(u)=\left(\Delta^{k} G^{m}(u)\right)_{B}$ almost everywhere in $B$. Thus $\triangle^{k} G^{m}(u)$ is a closed form and is a solution of $A$-harmonic equation (1.3). Hence Lemma 2.2 holds for $\Delta^{k} G^{m}(u)$. Replacing $u$ by $\triangle^{k} G^{m}(u)$ in Lemma 2.2, we have

$$
\left\|\Delta^{k} G^{m}(u)\right\|_{s, B} \leq C_{1}|B|^{(t-s) / s t}\left\|\Delta^{k} G^{m}(u)\right\|_{t, \sigma_{1} B},
$$


where $\sigma_{1}>1$ is a constant such that $\sigma_{1} B \subset M$. Since $|B|>d_{0}>0$, there exists a constant $C_{2}>0$ such that $\frac{1}{|B|^{1 / n}} \leq C_{2}$. Combining (2.15) and Theorem 2.5, we obtain

$$
\begin{aligned}
\left\|\Delta^{k} G^{m}(u)\right\|_{s, B} & \leq C_{1}|B|^{(t-s) / s t}\left\|\Delta^{k} G^{m}(u)\right\|_{t, \sigma_{1} B} \\
& \leq C_{3}|B|^{(t-s) / s t}\|u\|_{t, \sigma_{2} B} \\
& =C_{3}|B|^{\frac{1}{s}+\frac{1}{n}-\frac{1}{t}} \frac{1}{|B|^{1 / n}}\|u\|_{t, \sigma_{2} B} \\
& \leq C_{4}|B|^{\frac{1}{s}+\frac{1}{n}-\frac{1}{t}}\|u\|_{t, \sigma_{2} B},
\end{aligned}
$$

where $\sigma_{2}>\sigma_{1}>1$ with $\sigma_{2} B \subset M$.

(2) Second, if the the measure $\left|\left\{x \in B:\left|\triangle^{k} G^{m}(u)-\left(\triangle^{k} G^{m}(u)\right)_{B}\right|>0\right\}\right|>0$, then (2.1) holds for $\Delta^{k} G^{m}(u)$, and thus we have

$$
\left\|\Delta^{k} G^{m}(u)\right\|_{n t /(n-t), B} \leq C_{5}\left\|\Delta^{k} G^{m}(u)-\left(\triangle^{k} G^{m}(u)\right)_{B}\right\|_{n t /(n-t), B} .
$$

Note that $G \triangle(u)=\triangle G(u)$ and $k<m$. Then $d \triangle^{k} G^{m}(u)=d G\left(\triangle^{k} G^{m-1}(u)\right)$ and $k \leq m-1$. Thus, combining Lemma 2.1 and Theorem 2.5, we have

$$
\left\|d \triangle^{k} G^{m}(u)\right\|_{t, B}=\left\|d G\left(\triangle^{k} G^{m-1}(u)\right)\right\|_{t, B} \leq C_{6}\left\|\Delta^{k} G^{m-1}(u)\right\|_{t, \sigma_{3} B} \leq C_{7}\|u\|_{t, \sigma_{4} B},
$$

where $\sigma_{4}>\sigma_{3}>1$ are constants such that $\sigma_{4} B \subset M$. Since $1<t<n$, from Lemma 2.3 and (2.18) we have

$$
\begin{aligned}
& \left\|\Delta^{k} G^{m}(u)-\left(\triangle^{k} G^{m}(u)\right)_{B}\right\|_{n t /(n-t), B} \\
& \quad=\left(\int_{B}\left|\Delta^{k} G^{m}(u)-\left(\triangle^{k} G^{m}(u)\right)_{B}\right|^{n t /(n-t)} d x\right)^{(n-t) / n t} \\
& \quad \leq C_{8}\left(\int_{B}\left|d \triangle^{k} G^{m}(u)\right|^{t} d x\right)^{1 / t} \\
& \quad=C_{8}\left\|d \triangle^{k} G^{m}(u)\right\|_{t, B} \\
& \quad \leq C_{9}\|u\|_{t, \sigma_{4} B} .
\end{aligned}
$$

By the monotonicity property of the $L^{t}$-space, we obtain the inequality

$$
\left(\frac{1}{|B|} \int_{B}\left|\Delta^{k} G^{m}(u)\right|^{s} d x\right)^{1 / s} \leq\left(\frac{1}{|B|} \int_{B}\left|\Delta^{k} G^{m}(u)\right|^{n t /(n-t)} d x\right)^{(n-t) / n t}
$$

for any $s$ such that $0<s<n t /(n-t)$. Inequality (2.20) shows that

$$
\left\|\triangle^{k} G^{m}(u)\right\|_{s, B} \leq|B|^{1 / s+1 / n-1 / t}\left\|\Delta^{k} G^{m}(u)\right\|_{n t /(n-t), B} .
$$

Combining (2.21), (2.17), and (2.19), we have

$$
\left\|\Delta^{k} G^{m}(u)\right\|_{s, B} \leq C_{10}|B|^{1 / s+1 / n-1 / t}\|u\|_{t, \sigma_{4} B} .
$$


From (2.16) and (2.22) we get (2.14) for $1<t<n$. Thus we complete the proof of Theorem 2.6

In Theorem 2.6, since $1<t<n$, then $\frac{n t}{n-t} \rightarrow+\infty$ as $t \rightarrow n^{-}$. Since $0<s<n t /(n-t)$, $s$ can be greater than $t$, and thus the composite operator $\Delta^{k} G^{m}(u)$ has higher norm than the differential form $u$. Next, we prove (2.14) for $t \geq n$.

Theorem 2.7 Let $u \in L_{\text {loc }}^{t}\left(M, \Lambda^{l}\right)$ be a smooth differential form on $M$, G be Green's operator, and $\triangle$ be the Laplace-Beltrami operator on $M, l=1,2, \ldots, n, t \geq n$. Then $\triangle^{k} G^{m}(u) \in$ $L_{\text {loc }}^{s}\left(M, \Lambda^{l}\right)$ for any constant $s>0$. Moreover, there exists a constant $C$, independent of $u$, such that

$$
\left\|\triangle^{k} G^{m}(u)\right\|_{s, B} \leq C|B|^{1 / s+1 / n-1 / t}\|u\|_{t, \sigma B}
$$

for all balls $B$ with $\sigma B \subset M$ and $|B|>d_{0}$ and any positive integer $k<m$, where $\sigma>1$ and $d_{0}>0$ are constants.

Proof We prove this theorem in the following two cases: (1) First, if the measure $\mid\{x \in B$ : $\left.\left|\triangle^{k} G^{m}(u)-\left(\triangle^{k} G^{m}(u)\right)_{B}\right|>0\right\} \mid=0$, then applying the same method as in the proof of Theorem 2.6, we can prove (2.23) for any ball $B$ with $\sigma B \subset M$, where $\sigma>1$ is a constant.

(2) Next, let the measure $\left|\left\{x \in B:\left|\triangle^{k} G^{m}(u)-\left(\triangle^{k} G^{m}(u)\right)_{B}\right|>0\right\}\right|>0$. Select $p=$ $\max \{1, s / t\}$ and $r=\frac{n p t}{n+p t}$. Then $1<r<n$. Since $t \geq n$, that is, $n-t \leq 0$, we have $r-t=$ $\frac{t(p(n-t)-n)}{n+p t}<0$, that is, $r<t$. Since $1<r<n$, Lemma 2.3 holds for $\Delta^{k} G^{m}(u)$ and $r$. Thus replacing $u$ and $p$ by $\triangle^{k} G^{m}(u)$ and $r$, respectively, in Lemma 2.3, we have

$$
\left(\int_{B}\left|\Delta^{k} G^{m}(u)-\left(\Delta^{k} G^{m}(u)\right)_{B}\right|^{n r /(n-r)} d x\right)^{(n-r) / n r} \leq C_{1}\left(\int_{B}\left|d \Delta^{k} G^{m}(u)\right|^{r} d x\right)^{1 / r} .
$$

From Lemmas 2.4 and 2.1 and Theorem 2.5 we obtain

$$
\begin{aligned}
\left(\int_{B}\left|d \triangle^{k} G^{m}(u)\right|^{r} d x\right)^{1 / r} & =\left(\int_{B}\left|d G\left(\triangle^{k} G^{m-1}(u)\right)\right|^{r} d x\right)^{1 / r} \\
& \leq C_{2}\left(\int_{\sigma_{1} B}\left|\triangle^{k} G^{m-1}(u)\right|^{r} d x\right)^{1 / r} \\
& \leq C_{3}\left(\int_{\sigma_{2} B}|u|^{r} d x\right)^{1 / r}
\end{aligned}
$$

for all balls $B$ with $\sigma_{2} B \subset M$, where $\sigma_{2}>\sigma_{1}>1$. Since $r<t$, applying the monotonicity property of the $L^{t}$ space, we have

$$
\left(\frac{1}{\left|\sigma_{2} B\right|} \int_{\sigma_{2} B}|u|^{r} d x\right)^{1 / r} \leq\left(\frac{1}{\left|\sigma_{2} B\right|} \int_{\sigma_{2} B}|u|^{t} d x\right)^{1 / t}
$$

Inequality (2.26) is equivalent to

$$
\left(\int_{\sigma_{2} B}|u|^{r} d x\right)^{1 / r} \leq C_{4}|B|^{1 / r-1 / t}\left(\int_{\sigma_{2} B}|u|^{t} d x\right)^{1 / t}
$$


Since the measure $\left|\left\{x \in B:\left|\triangle^{k} G^{m}(u)-\left(\triangle^{k} G^{m}(u)\right)_{B}\right|>0\right\}\right|>0$, estimates (2.1) hold for $\triangle^{k} G^{m}(u)$. In the second half of (2.1), we replace $u$ and $s$ by $\triangle^{k} G^{m}(u)$ and $n r /(n-r)$ :

$$
\begin{aligned}
& \left(\int_{B}\left|\triangle^{k} G^{m}(u)\right|^{n r /(n-r)} d x\right)^{(n-r) / n r} \\
& \quad \leq C_{5}\left(\int_{B}\left|\triangle^{k} G^{m}(u)-\left(\triangle^{k} G^{m}(u)\right)_{B}\right|^{n r /(n-r)} d x\right)^{(n-r) / n r} .
\end{aligned}
$$

Since $p=\max \{1, s / t\}$, we have $p t \geq s$. Since $n r /(n-r)=p t$, we have $n r /(n-r) \geq s$, and thus applying the monotonicity property of the $L^{t}$ space, we have

$$
\left(\frac{1}{|B|} \int_{B}\left|\triangle^{k} G^{m}(u)\right|^{s} d x\right)^{1 / s} \leq\left(\frac{1}{|B|} \int_{B}\left|\Delta^{k} G^{m}(u)\right|^{n r /(n-r)} d x\right)^{(n-r) / n r} .
$$

Combining (2.29), (2.28), (2.24), (2.25), and (2.27), we obtain

$$
\begin{aligned}
& \left(\int_{B}\left|\Delta^{k} G^{m}(u)\right|^{s} d x\right)^{1 / s} \\
& \quad \leq|B|^{1 / s-(n-r) / n r}\left(\int_{B}\left|\Delta^{k} G^{m}(u)\right|^{n r /(n-r)} d x\right)^{(n-r) / n r} \\
& \quad \leq C_{5}|B|^{1 / s-(n-r) / n r}\left(\int_{B}\left|\Delta^{k} G^{m}(u)-\left(\Delta^{k} G^{m}(u)\right)_{B}\right|^{n r /(n-r)} d x\right)^{(n-r) / n r} \\
& \quad \leq C_{6}|B|^{1 / s-(n-r) / n r+1 / r-1 / t}\left(\int_{\sigma_{2} B}|u|^{t} d x\right)^{1 / t} \\
& \quad=C_{6}|B|^{1 / s+1 / n-1 / t}\left(\int_{\sigma_{2} B}|u|^{t} d x\right)^{1 / t} \cdot
\end{aligned}
$$

Inequality (2.30) implies (2.23). Therefore we complete the proof of this theorem.

In Theorem 2.7, from the condition $t \geq n$ we have $\frac{1}{s}+\frac{1}{n}-\frac{1}{t}>0$, which is also presented in Theorem 2.6, and thus combining Theorems 2.6 and 2.7, we easily obtain the following theorem for any $t>1$.

Theorem 2.8 Let $u \in L_{\mathrm{loc}}^{t}\left(M, \Lambda^{l}\right)$ be a smooth differential form defined on $M, G$ be Green's operator, and $\triangle$ be the Laplace-Beltrami operator defined on $M, l=1,2, \ldots, n, t>1$. Then $\triangle^{k} G^{m}(u) \in L_{\mathrm{loc}}^{s}\left(M, \Lambda^{l}\right)$ for any constant $s>0$ such that $\frac{1}{s}+\frac{1}{n}-\frac{1}{t}>0$. Moreover, there exists a constant $C$, independent of $u$, such that

$$
\left\|\Delta^{k} G^{m}(u)\right\|_{s, B} \leq C|B|^{1 / s+1 / n-1 / t}\|u\|_{t, \sigma B}
$$

for all balls $B$ with $\sigma B \subset M$ and $|B|>d_{0}$ and any positive integer $k<m$, where $\sigma>1$ and $d_{0}>0$ are constants.

\section{Global higher norm inequalities for composite operator $\Delta^{k} G^{m}$}

In this section, based on Theorem 2.8, we will prove the global higher norm estimate for the composite operator $\triangle^{k} G^{m}(u)$ in any bounded domain $M \subset R^{n}$. Then we will establish the corresponding global higher norm estimate with Radon measure. In the following 
proof of related theorems, we need the following modified Whitney cover in [25]; see [23] for more detail about Whitney covers.

Lemma 3.1 Each $\Omega \subset R^{n}$ has a modified Whitney cover of cubes $W=\left\{Q_{i}\right\}$ that satisfy

$$
\begin{aligned}
& \bigcup_{i} Q_{i}=\Omega, \\
& \sum_{Q \in W} \chi_{\sqrt{5 / 4} Q} \leq N \cdot \chi_{\Omega}
\end{aligned}
$$

for all $x \in R^{n}$ and some $N>1$, and if $Q_{i} \cap Q_{j} \neq \emptyset$, then there exists a cube $R$ in $Q_{i} \cap Q_{j}$ such that $Q_{i} \cup Q_{j} \subset N R$. Moreover, if $\Omega$ is a $\delta$-John, then there is a distinguished cube $Q_{0} \in W$ that can be connected with every cube $Q \in W$ by a chain of cubes $Q_{0}, Q_{1}, \ldots, Q_{k}$ from $W$ and such that $Q \subset \rho Q_{i}, i=1,2, \ldots, k$, for some $\rho=\rho(n, \delta)$.

Now we will give the global higher norm inequality for the composite operator $\triangle^{k} G^{m}(u)$ based on Theorem 2.8 .

Theorem 3.2 Let $u \in L_{\mathrm{loc}}^{t}\left(M, \Lambda^{l}\right)$ be a smooth differential form defined on a bounded domain $M, G$ be Green's operator, and $\triangle$ be the Laplace-Beltrami operator, $l=1,2, \ldots, n$, $t>1$. Then $\triangle^{k} G^{m}(u) \in L_{\mathrm{loc}}^{s}\left(M, \Lambda^{l}\right)$ for any constant $s>0$ such that $\frac{1}{s}+\frac{1}{n}-\frac{1}{t}>0$. Moreover, there exists a constant $C$, independent of $u$, such that

$$
\left\|\triangle^{k} G^{m}(u)\right\|_{s, M} \leq C|M|^{1 / s+1 / n-1 / t}\|u\|_{t, M}
$$

for any positive integer $k<m$.

Proof From Lemma 3.1, we know that there exists a sequence of cubes $W=\left\{B_{i}\right\}$ such that $\bigcup_{i} B_{i}=M$ and $\sum_{B_{i} \in W} \chi_{\sqrt{5 / 4} B_{i}} \leq N \cdot \chi_{M}(x)$ for all $x \in M$, where $N>1$ is some constant. Hence, for $u \in L_{\mathrm{loc}}^{t}\left(M, \Lambda^{l}\right)$, using Theorem 2.8, we have

$$
\begin{aligned}
\left\|\Delta^{k} G^{m}(u)\right\|_{s, M} & \leq \sum_{B_{i} \in W}\left\|\Delta^{k} G^{m}(u)\right\|_{s, B_{i}} \\
& \leq \sum_{B_{i} \in W} C_{1}\left|B_{i}\right|^{1 / s+1 / n-1 / t}\|u\|_{t, \sigma B_{i}} \\
& \leq C_{1}|M|^{1 / s+1 / n-1 / t} \sum_{B_{i} \in W}\left(\int_{\sigma B_{i}}|u|^{t} d x\right)^{1 / t} \\
& =C_{1}|M|^{1 / s+1 / n-1 / t} \sum_{B_{i} \in W}\left(\int_{M}|u|^{t} d x \cdot \chi_{\sigma B_{i}}\right)^{1 / t} \\
& =C_{1}|M|^{1 / s+1 / n-1 / t} N \cdot\left(\int_{M}|u|^{t} d x\right)^{1 / t} \\
& =C_{1} N|M|^{1 / s+1 / n-1 / t}\|u\|_{t, M} \\
& =C_{2}|M|^{1 / s+1 / n-1 / t}\|u\|_{t, M},
\end{aligned}
$$


where $C_{2}=C_{1} N$ is independent of $u$ and all $B_{i}$. Thus we complete the proof of Theorem 3.2.

In Theorem 3.2, if we assume that $s>t$, then Theorem 3.2 reduces to the global higher norm estimate for composite operator $\Delta^{k} G^{m}$. Next, we consider the following global norm comparison equipped with Radon measure based on Theorem 3.2.

Theorem 3.3 Let $u \in L_{\mathrm{loc}}^{t}\left(M, \Lambda^{l}\right)$ be a smooth differential form defined on a bounded domain $M, G$ be Green's operator, $\triangle$ be the Laplace-Beltrami operator, and $\hbar_{1}(y)$ and $\hbar_{2}(y)$ be two continuous nonnegative functions defined on $(0,+\infty)$ with conditions: (1) $\lim _{y \rightarrow 0} \hbar_{1}(y)=$ 0 ; (2) $\lim _{y \rightarrow 0} \hbar_{2}(y)=\infty, l=1,2, \ldots, n, t>1$. Then $\Delta^{k} G^{m}(u) \in L_{\mathrm{loc}}^{s}\left(M, \Lambda^{l}\right)$ for any constant $s>0$ such that $\frac{1}{s}+\frac{1}{n}-\frac{1}{t}>0$. Moreover, there exists a constant $C$, independent of $u$, such that

$$
\left\|\Delta^{k} G^{m}(u)\right\|_{s, M, v_{1}} \leq C|M|^{1 / s+1 / n-1 / t}\|u\|_{t, M, v_{2}}
$$

for any positive integer $k<m$ and the Radon measure $v_{1}, v_{2}$ defined by

$$
d v_{1}=\hbar_{1}(\operatorname{dist}(x, \partial M)) d x, \quad d v_{2}=\hbar_{2}(\operatorname{dist}(x, \partial M)) d x .
$$

Proof From Theorem 3.2, we know that there exists a constant $C_{1}$, independent of $u$, such that

$$
\left\|\Delta^{k} G^{m}(u)\right\|_{s, M} \leq C_{1}|M|^{1 / s+1 / n-1 / t}\|u\|_{t, M} .
$$

Since $\lim _{y \rightarrow 0} \hbar_{1}(y)=0$, for any small positive number $\epsilon$, there exists $\delta(\epsilon)>0$ such that $\hbar_{1}(\operatorname{dist}(x, \partial M))<\epsilon$ for all $x \in M$ with $\operatorname{dist}(x, \partial M)<\delta$. Let $M^{\prime}=\{x \in M$, dist $(x, \partial M)<\delta\}$ and $M^{\prime \prime}=M-M^{\prime}$. Then for all $x \in M^{\prime \prime}$, we have

$$
\delta \leq \operatorname{dist}(x, \partial M)<\operatorname{diam}(M) .
$$

Therefore by the continuity and nonnegativity of $\hbar_{1}$ we have that there exists $C_{2}>0$ such that

$$
0<\hbar_{1}(\operatorname{dist}(x, \partial M))<C_{2}
$$

for all $x \in M^{\prime \prime}$. Thus we have

$$
\begin{aligned}
& \left\|\Delta^{k} G^{m}(u)\right\|_{s, M, v_{1}} \\
& \quad=\left(\int_{M}\left|\Delta^{k} G^{m}(u)\right|^{s} \cdot \hbar_{1}(\operatorname{dist}(x, \partial M)) d x\right)^{\frac{1}{s}} \\
& \quad \leq\left(\epsilon \int_{M^{\prime}}\left|\Delta^{k} G^{m}(u)\right|^{s} d x+C_{2} \int_{M^{\prime \prime}}\left|\Delta^{k} G^{m}(u)\right|^{s} d x\right)^{\frac{1}{s}} \\
& \quad \leq C_{3}\left(\int_{M}\left|\Delta^{k} G^{m}(u)\right|^{s} d x\right)^{\frac{1}{s}},
\end{aligned}
$$


where $C_{3}=\max \left\{\epsilon^{\frac{1}{s}}, C_{2}^{\frac{1}{s}}\right\}$. Combining (3.5) and (3.4), we have

$$
\left\|\Delta^{k} G^{m}(u)\right\|_{s, M, v_{1}} \leq C_{3}\left\|\Delta^{k} G^{m}(u)\right\|_{s, M} \leq C_{4}|B|^{1 / s+1 / n-1 / t}\|u\|_{t, M} .
$$

Note that $\lim _{y \rightarrow 0} \frac{1}{\hbar_{2}(y)}=0$. Then there exists $\delta_{1}(\epsilon)>0$ such that $\frac{1}{\hbar_{2}(\operatorname{dist}(x, \partial M))}<\epsilon$ for all $x \in M$ with $\operatorname{dist}(x, \partial M)<\delta_{1}$. Let $M_{1}^{\prime}=\left\{x \in M\right.$, $\left.\operatorname{dist}(x, \partial M)<\delta_{1}\right\}$ and $M_{1}^{\prime \prime}=M-M_{1}^{\prime}$. Then for all $x \in M_{1}^{\prime \prime}$, we have

$$
\delta_{1} \leq \operatorname{dist}(x, \partial M)<\operatorname{diam}(M) .
$$

Therefore by the continuity and nonnegativity of $\hbar_{2}$ we have that there exists a constant $C_{5}>0$ such that

$$
0<\frac{1}{\hbar_{2}(\operatorname{dist}(x, \partial M))}<C_{5}
$$

for all $x \in M_{1}^{\prime \prime}$. Therefore we obtain

$$
\begin{aligned}
\|u\|_{t, M} & =\left(\int_{M}|u|^{t} \frac{1}{\hbar_{2}(\operatorname{dist}(x, \partial M))} d v_{2}\right)^{\frac{1}{t}} \\
& \leq\left(\epsilon \int_{M_{1}^{\prime}}|u|^{t} d v_{2}+C_{5} \int_{M_{1}^{\prime \prime}}|u|^{t} d v_{2}\right)^{\frac{1}{t}} \\
& \leq C_{6}\left(\int_{M}|u|^{t} d \nu_{2}\right)^{\frac{1}{t}}=C_{6}\|u\|_{t, M, \nu_{2}}
\end{aligned}
$$

where $C_{6}=\max \left\{\epsilon^{\frac{1}{t}}, C_{5}^{\frac{1}{t}}\right\}$. By (3.6) and (3.7) we have

$$
\left\|\triangle^{k} G^{m}(u)\right\|_{s, M, \nu_{1}} \leq C_{7}|M|^{1 / s+1 / n-1 / t}\|u\|_{t, M, \nu_{2}}
$$

where $C_{7}$ is independent of $u$. Thus we complete the proof of Theorem 3.3.

In Theorem 3.3, choosing $\hbar_{1}(y)=y^{p}$ and $\hbar_{2}(y)=y^{-q}, 0<p, q<\infty$, we easily obtain the following corollary.

Corollary 3.4 Let $u \in L_{\mathrm{loc}}^{t}\left(M, \Lambda^{l}\right)$ be a smooth differential form defined on a bounded domain $M, G$ be Green's operator, and $\triangle$ be the Laplace-Beltrami operator, $l=1,2, \ldots, n$, $t>1$. Then $\triangle^{k} G^{m}(u) \in L_{\mathrm{loc}}^{s}\left(M, \Lambda^{l}\right)$ for any constant $s>0$. Moreover, there exists a constant $C$, independent of $u$, such that

$$
\begin{aligned}
& \left(\int_{M}\left|\Delta^{k} G^{m}(u)\right|^{s} \cdot(\operatorname{dist}(x, \partial M))^{p} d x\right)^{1 / s} \\
& \quad \leq C|M|^{1 / s+1 / n-1 / t}\left(\int_{M}|u|^{t} \frac{1}{(\operatorname{dist}(x, \partial M))^{q}} d x\right)^{1 / t}
\end{aligned}
$$

for any positive integer $k<m$ and any real numbers $0<p, q<\infty$. 


\section{Applications}

In many cases, it is very difficult to give the norm estimate for a composite operator. In this section, we give two examples to obtain the upper bounds for the norm of the composite operator $\triangle^{k} G^{m}(u)$ as applications of Theorem 3.2.

Example 4.1 Let $M=\left\{\left(x_{1}, x_{2}\right): x_{1}^{2}+x_{2}^{2} \leq a^{2}\right\} \subset R^{2}$, and let $u$ be the differential 1-form

$$
u\left(x_{1}, x_{2}\right)=x_{1} d x_{1}-x_{2} d x_{2}
$$

defined on $M$. Then $|M|=\pi a^{2}$, and $u$ is a differential form satisfying the conditions of Theorem 3.2. Thus we can estimate $\left\|\triangle^{k} G^{m}(u)\right\|_{s, M}$ in terms of $\|u\|_{t, M}$, where $s, t$ are two independent positive real numbers such that $t>1$ and $1 / s+1 / n-1 / t>0$. For any $\left(x_{1}, x_{2}\right) \in$ $M$, we have $|u|=\sqrt{x_{1}^{2}+x_{2}^{2}}$. By polar coordinate transformation $x_{1}=\rho \cos \theta, x_{2}=\rho \sin \theta$ we obtain

$$
\|u\|_{t, M}=\left(\int_{M}|u|^{t} d x\right)^{1 / t}=\left(\int_{0}^{2 \pi} d \theta \int_{0}^{a} \rho^{t+1} d \rho\right)^{1 / t}=\left(\frac{2 \pi a^{t+2}}{t+2}\right)^{1 / t} .
$$

Since $n=2$, for any $s>0$ such that $1 / s+1 / n-1 / t>0$, by Theorem 3.2 we have

$$
\begin{aligned}
\left\|\triangle^{k} G^{m}(u)\right\|_{s, M} & \leq C|M|^{1 / s+1 / n-1 / t}\|u\|_{t, M} \\
& =C\left(\pi a^{2}\right)^{1 / s+1 / 2-1 / t}\left(\frac{2 \pi a^{t+2}}{t+2}\right)^{1 / t} \\
& =C\left(\pi a^{2}\right)^{1 / s+1 / 2}\left(\frac{2 a^{t}}{t+2}\right)^{1 / t}
\end{aligned}
$$

for any positive integer $k<m$. Moreover, if we assume that $s=1 / 2$ and $t=1$ in (4.3), then $1 / s+1 / n-1 / t>0$, satisfying the condition of Theorem 3.2 , and thus we have

$$
\left\|\triangle^{k} G^{m}(u)\right\|_{1 / 2, M} \leq C\left(\frac{2}{3} \pi a^{3}\right)
$$

Example 4.2 Let $M=\left\{\left(x_{1}, x_{2}, x_{3}\right): x_{1}^{2}+x_{2}^{2}+x_{3}^{2} \leq a^{2}\right\} \subset R^{3}$ and $u\left(x_{1}, x_{2}, x_{3}\right)$ be a differential 2-form on $M$ defined by

$$
\begin{aligned}
u= & \frac{x_{1}}{\sqrt{x_{1}^{2}+x_{2}^{2}+x_{3}^{2}}} d x_{2} \Lambda d x_{3}-\frac{x_{2}}{\sqrt{x_{1}^{2}+x_{2}^{2}+x_{3}^{2}}} d x_{1} \Lambda d x_{3} \\
& +\frac{x_{3}}{\sqrt{x_{1}^{2}+x_{2}^{2}+x_{3}^{2}}} d x_{1} \Lambda d x_{2} .
\end{aligned}
$$

Then $|M|=\frac{4}{3} \pi a^{3}$ and $|u|=1$, and thus we obtain

$$
\|u\|_{t, M}=\left(\int_{M}|u|^{t} d x\right)^{1 / t}=\left(\int_{M} d x\right)^{1 / t}=|M|^{1 / t}=\left(\frac{4}{3} \pi a^{3}\right)^{1 / t}
$$

for any $t>1$. Applying Theorem 3.2, we have $\Delta^{k} G^{m}(u) \in L_{\text {loc }}^{s}(M)$ for any constant $s>0$ such that $1 / s+1 / n-1 / t>0$. Thus we can further obtain the following upper bound for the 
norm of the composite operator $\Delta^{k} G^{m}(u)$ :

$$
\begin{aligned}
\left\|\triangle^{k} G^{m}(u)\right\|_{s, M} & \leq C|M|^{1 / s+1 / n-1 / t}\|u\|_{t, M} \\
& =C\left(\frac{4}{3} \pi a^{3}\right)^{1 / s+1 / 3-1 / t}\left(\frac{4}{3} \pi a^{3}\right)^{1 / t} \\
& =C\left(\frac{4}{3} \pi a^{3}\right)^{1 / s+1 / 3}
\end{aligned}
$$

for any positive integer $k<m$.

Remark Examples 4.1 and 4.2 can be generalized to the $n$-dimensional space.

\section{Conclusion}

In this paper, we first give the local $L^{s}$ norm estimate for the composite operators $\triangle^{k} G^{m}(u)$ in terms of the $L^{s}$ norm of $u$ with commuting Laplace-Beltrami operator $\triangle$ and Green's operator $G$. At the same time, we also obtain the local and global $L^{s}$ norms of $\triangle^{k} G^{m}(u)$ in terms of the $L^{t}$ norm of differential forms $u$ for any constant $s>0$ such that $\frac{1}{s}+\frac{1}{n}-$ $\frac{1}{t}>0$. Then we establish a global higher norm estimate with Radon measure for composite operators $\triangle^{k} G^{m}$. At last, as applications of these results, we give two examples to estimate the higher norm of $\triangle^{k} G^{m}(u)$.

\section{Acknowledgements}

The authors wish to express their gratitude to the anonymous referees for their thoughtful suggestions, which have helped them to substantially improve the paper. They have thus been able to eliminate some mistakes and to present the manuscript in a better manner. The authors are also thankful to all editorial board members in Journal of Inequalities and Applications.

Funding

This research was supported by NNSF of China (No. 11961030) and by the Foundation of Jiangxi Provincial Education Department of China (Nos. GJJ191244, GJJ180446)

\section{Availability of data and materials}

Not applicable.

\section{Competing interests}

The authors declare that they have no competing interests.

\section{Authors' contributions}

Both authors jointly contributed to the main results, and HL drafted the manuscript. Both authors read and approved the final manuscript.

\section{Authors' information}

HL(1985-), male, lecturer, major in harmonic analysis; QL(1987-), female, lecturer, major in functional analysis.

\section{Author details}

'School of Science, JiangXi University of Science and Technology, Ganzhou, China. ${ }^{2}$ Department of Mathematics, Ganzhou Teachers College, Ganzhou, China.

\section{Publisher's Note}

Springer Nature remains neutral with regard to jurisdictional claims in published maps and institutional affiliations.

Received: 9 September 2019 Accepted: 7 April 2020 Published online: 16 April 2020

\section{References}

1. Xing, Y.M., Ding, S.S.: Norms of the composition of the maximal and projection operators. Nonlinear Anal. 72 4614-4624 (2010). https://doi.org/10.1016/j.na.2010.02.038

2. $\mathrm{Bi}, \mathrm{H}$. : Weighted inequalities for potential operators on differential forms. J. Inequal. Appl. 2010, Article ID 713625 (2010). https://doi.org/10.1155/2010/713625 
3. Xing, Y.M.: Two-weight embedding inequalities for solutions to the A-harmonic equation. J. Math. Anal. Appl. 307 555-564 (2005). https://doi.org/10.1016/j.jmaa.2005.03.019

4. Ding, S.S., Sylvester, D.: Weak reverse Hölder inequalities and imbedding inequalities for solutions to the A-harmonic equation. Nonlinear Anal., Theory Methods Appl. 51, 783-800 (2002).

https://doi.org/10.1016/S0362-546X(01)00862-8

5. Scott, C.: $L^{p}$-theory of differntial forms on manifolds. Trans. Am. Math. Soc. 347, 2075-2096 (1995). https://doi.org/10.2307/2154923

6. Dai, Z.M., Li, H.C., Li, Q.F.: Inequalities for the fractional convolution operator on differential forms. J. Inequal. Appl. 2018, Article ID 176 (2018). https://doi.org/10.1186/s13660-018-1768-x

7. Li, H.C., Li, Q.F.: Some weighted norm estimates for the composition of the homotopy and Green's operator. Abstr. Appl. Anal. 2014, Article ID 941658 (2014). https://doi.org/10.1155/2014/941658

8. Xing, Y.M., Ding, S.S.: Caccioppoli inequalities with Orlicz norms for solutions of harmonic equations and applications. Nonlinearity 23, 1109-1119 (2010). https://doi.org/10.1088/0951-7715/23/5/005

9. Ding, S.S.: $L^{\varphi}(\mu)$-averaging domains and the quasihyperbolic metric. Comput. Math. Appl. 47, 1611-1618 (2004). https://doi.org/10.1016/i.camwa.2004.06.016

10. Mishra, L.N.: On existence and behavior of solutions to some nonlinear integral equations with applications. Ph.D. thesis, National Institute of Technology, Silchar 788 010, Assam, India (2017)

11. Mishra, V.N., Khatri, K., Mishra, L.N., Deepmala: Inverse result in simultaneous approximation by Baskakov-Durrmeyer-Stancu operators. J. Inequal. Appl. 2013, Article ID 586 (2013). https://doi.org/10.1186/1029-242X-2013-586

12. Sbordone, C.: Higher integrability of the gradient of minimizers of functionals with nonstandard growth conditions. Commun. Pure Appl. Math. 43, 673-683 (1990). https://doi.org/10.1002/cpa.3160430505

13. Gehring, F.W.: The $L^{P}$-integrability of partial derivatives of a quasiconforming mappings. Acta Math. 130, 265-277 (1973)

14. Strdulinsky, E.W.: Higher integrability from reverse Hölder inequalities. Indiana Univ. Math. J. 29, 407-413 (1980). https://doi.org/10.1512/iumj.1980.29.29029

15. Weigert, S., Müller, G.: Quantum integrability and action operators in spin dynamics. Chaos Solitons Fractals 5 , 1419-1438 (1995). https://doi.org/10.1016/0960-0779(95)00021-U

16. Mishra, V.N.: Some problems on approximations of functions in Banach spaces. Ph.D. thesis, Indian Institute of Technology, Roorkee 247 667, Uttarakhand, India (2007)

17. Gairola, A.R., Deepmala, Mishra, L.N.: On the $q$-derivatives of a certain linear positive operators. Iran. J. Sci. Technol. Trans. A, Sci. 42(3), 1409-1417 (2018). https://doi.org/10.1007/s40995-017-0227-8

18. Gandhi, R.B., Deepmala, Mishra, V.N.: Local and global results for modified Szász-Mirakjan operators. Math. Methods Appl. Sci. 40(7), 2491-2504 (2017). https://doi.org/10.1002/mma.4171

19. Agarwal, R.P., Ding, S.S., Nolder, C.A.: Inequalities for Differential Forms. Springer, New York (2009)

20. Ding, S.S., Shi, G.N., Xing, Y.M.: Higher integrability of iterated operators on differential forms. Nonlinear Anal. 145, 83-96 (2016). https://doi.org/10.1016/j.na.2016.07.012

21. Xing, Y.M., Ding, S.S.: Higher integrability of Green's operator and homotopy operator. J. Math. Anal. Appl. 446 648-662 (2017). https://doi.org/10.1016/j.jmaa.2016.09.007

22. Ding, S.S., Liu, B.: Norm inequalities for composition of Dirac and Green's operators. J. Inequal. Appl. 2013, Article ID 436 (2013). https://doi.org/10.1186/1029-242x-2013-436

23. Stein, E.M.: Singular Integrals and Differentiability Properties of Functions. Princeton University Press, Princeton (1970)

24. Iwaniec, T., Lutoborski, A.: Integral estimates for null Lagrangians. Arch. Ration. Mech. Anal. 125, 25-79 (1993). https://doi.org/10.1007/BF00411477

25. Nolder, C.A.: Hardy-Littlewood theorems for A-harmonic tensors. III. J. Math. 43, 613-631 (1999)

\section{Submit your manuscript to a SpringerOpen ${ }^{\circ}$ journal and benefit from:}

- Convenient online submission

- Rigorous peer review

- Open access: articles freely available online

- High visibility within the field

- Retaining the copyright to your article

Submit your next manuscript at $\gg$ springeropen.com 